\title{
FUNDAMENTAL PROPERTIES OF HAMILTONIAN OPERATORS OF SCHRÖDINGER TYPE
}

\author{
BY \\ TOSIO KATO
}

Introduction. The fundamental quality required of operators representing physical quantities in quantum mechanics is that they be hypermaximal( $\left.{ }^{1}\right)$ or self-adjoint $\left({ }^{2}\right)$ in the strict sense employed in the theory of Hilbert space, which is equivalent to saying that the eigenvalue problem is completely solvable for them, that is, that there exists a complete set (discrete or continuous) of eigenfunctions. It will, therefore, be one of the important problems of mathematical physics to show that this is actually the case for all operators appearing in current structure of quantum mechanics, for the self-adjointness of an operator is a property requiring careful separate consideration and by no means a direct consequence of its Hermitian symmetry [cf. (S), chap. X].

The problem has, of course, been solved in the case of operators for which the eigenvalue problem is explicitly solved by separation of variables or other methods, but it seems not to have been settled in the general case of manyparticle systems $\left({ }^{3}\right)$.

The main purpose of the present paper is to show that the Schrödinger Hamiltonian operator $\left(^{4}\right)$ of every atom, molecule, or ion, in short, of every system composed of a finite number of particles interacting with each other through a potential energy, for instance, of Coulomb type, is essentially selfadjoint $\left({ }^{5}\right)\left({ }^{6}\right)$. Thus our result serves as a mathematical basis for all theoretical works concerning nonrelativistic quantum mechanics, for they always pre-

Received by the editors October 15, 1948.

(1) J. v. Neumann, Mathematische Grundlagen der Quantenmechanik, Berlin, Springer, 1932, p. 88. This book will be referred to in the following as $(\mathrm{N})$.

$\left(^{2}\right)$ M. H. Stone, Linear transformations in Hilbert space, Amer. Math. Soc. Colloquium Publications, vol. 15, New York, 1932, chaps. IV, V. This book will be referred to as (S).

(3) E. C. Kemble, The fundamental principles of quantum mechanics, International Series in Physics, New York and London, 1937, p. 196. This book will be referred to as (K).

$\left.{ }^{4}\right)$ The spin-interaction is neglected in the following. In consequence, we need not take into account the spin and symmetry property of wave functions, for their effects consist solely in making the product of the Hilbert space $\mathfrak{S}$ of scalar wave functions with the finite-dimensional spin space and then decomposing the product space according to symmetry property; but the essentially-self-adjointness of our operator once established in $\mathfrak{E}$ is preserved throughout this procedure.

(5) A symmetric operator is said to be essentially self-adjoint if its closure is self-adjoint. See (S), pp. 51, 343 .

(6) This is noteworthy because there are potentials for which the statement is false. A simple example is a negative inverse-square potential. The allowable singularity will be specified below. 
suppose, at least tacitly, the self-adjointness of Hamiltonian operators.

It will be better at this point to comment on the terms "essentially selfadjoint." The Hamiltonian operator of a system composed of $s+1$ particles has the form

$$
-\sum_{i=0}^{s}\left(h^{2} / 8 \pi^{2} m_{i}\right) \operatorname{grad}_{i}^{2}+V\left(\boldsymbol{r}_{0}, \boldsymbol{r}_{1}, \cdots, \boldsymbol{r}_{s}\right),
$$

where $\boldsymbol{r}_{i}=\left(x_{i}, y_{i}, z_{i}\right)$ is the position vector of the $i$ th particle. If we assume that the potential energy $V$ is dependent only on relative position of particles, the translation of the whole system can be separated out, and the remaining relative motion is, after suitable change of unit of length, governed by the operator of the form $[(\mathrm{K})$, p. 196]

$$
H=-\sum_{i=1}^{s} \mu_{i} \operatorname{grad}_{i}^{2}-\mu_{0}\left(\sum_{i=1}^{s} \operatorname{grad}_{i}\right)^{2}+V\left(\boldsymbol{r}_{1}, \cdots, \boldsymbol{r}_{s}\right),
$$

where $\mu_{0}, \mu_{1}, \cdots, \mu_{s}$ are positive constants. If we allow $\mu_{0}$ to vanish, (2) contains (1) as a special case, so that we shall in the following adopt (2) as the Hamiltonian of our system and assume $\mu_{0} \geqq 0$.

Now a differential operator is generally a troublesome one because its domain is only vaguely defined [cf. (S), p. 113]. Questions like these remain open: Must the wave functions on which it operates have everywhere continuous derivatives? Or what degree of singularity is permitted them at points where the potential is singular? How rapidly must they vanish at infinity? There seems to have been no agreement between different authors on these "boundary conditions" $\left.{ }^{7}\right)$. These difficulties will ultimately disappear as a natural consequence of our investigation, but at present we emphasize the fact that in general the domain of a differential operator is by no means unambiguously prescribed. It is obvious from the outset that we cannot expect such an obscure operator to be self-adjoint in the literal sense.

We must, therefore, start by restricting the domain of the given differential operator $H$ to sufficiently regular functions, and then appropriately extend the operator thus defined so as to become self-adjoint. On the other hand, however, we know that $H$ can certainly be extended to a self-adjoint operator, for $H$ is (Hermitian) symmetric and real [(N), p. 88; (S), p. 361].

Thus the kernel of our problem lies in the fact that $H$ has only one selfadjoint extension unless its domain is too artificially restricted. This is exactly what is meant by saying that $H$ is essentially self-adjoint. If this were not the case, $H$ could be extended in infinitely many ways [(N), p. 87; $(\mathrm{S})$, p. 361] to a self-adjoint operator, and would lead to different spectra according to different and more or less arbitrary choices of boundary conditions; it

(7) Cf. (K), pp. 79, 197; W. Pauli, Handbuch der Physik, 24/1, 2d ed., p. 121; G. Jaffé, Zeitschrift für Physik vol. 66 (1930) p. 770, where other literature can be found. 
would mean that $H$ is an incomplete operator from the standpoint of quantum mechanics.

We impose on $H$ the following minimum requirements, which are to express that its domain is not too artificially restricted.

(i) $H$ is a (Hermitian) symmetric linear operator;

(ii) $H$ can be applied on all functions of the form

$$
g(\boldsymbol{r})=P\left(\boldsymbol{r}_{1}, \cdots, \boldsymbol{r}_{s}\right) \exp \left\{-(1 / 2)\left(\boldsymbol{r}_{1}^{2}+\cdots+\boldsymbol{r}_{s}^{2}\right)\right\},
$$

where $P$ is a polynomial of $3 s$ coordinates. Of course, (ii) implies some restriction on the potential energy $V$, for $V g$ must belong to the Hilbert space $\mathfrak{S}=L_{2}$ defined over the whole $3 s$-dimensional space $\left(\boldsymbol{r}_{1}, \cdots, \boldsymbol{r}_{s}\right)$. This is guaranteed by the following assumptions for $V$, which are made throughout this paper. $V$ shall be expressible as

$$
V\left(\boldsymbol{r}_{1}, \cdots, \boldsymbol{r}_{s}\right)=V^{\prime}\left(\boldsymbol{r}_{1}, \cdots, \boldsymbol{r}_{8}\right)+\sum_{i=1}^{s} V_{0 i}\left(\boldsymbol{r}_{i}\right)+\sum_{i<j}^{1, s} V_{i j}\left(\boldsymbol{r}_{i}-\boldsymbol{r}_{j}\right),
$$

where $V^{\prime}$ is bounded in the whole configuration space, and $V_{i j}(0 \leqq i<j \leqq s)$, all defined in the 3-dimensional space, are locally square-integrable and bounded at infinity. In other words, we assume that there are two constants $R, C$ such that

$$
\begin{aligned}
\left|V^{\prime}\left(\boldsymbol{r}_{1}, \cdots, \boldsymbol{r}_{s}\right)\right| & \leqq C, \\
\int_{r \leqq R}\left|V_{i j}(x, y, z)\right|^{2} d x d y d z \leqq C^{2}, & \\
\left|V_{i j}(x, y, z)\right| \leqq C(r>R) & (0 \leqq i<j \leqq s) . \\
& \left(r=\left(x^{2}+y^{2}+z^{2}\right)^{1 / 2}\right) .
\end{aligned}
$$

It is easily verified that these assumptions are satisfied by Coulomb potentials, or potentials of the form $r^{-m}$ if $m<3 / 2$.

We need not specify the domain of $H$ in detail; we have only to admit that $H$ is defined on a certain domain $\mathcal{D}_{H}$ such that (i) and (ii) are satisfied. If we denote by $\mathcal{D}_{1}$ the set of all functions (3), (ii) means that $\mathcal{D}_{H} \supseteq \mathcal{D}_{1}$. Let $H_{1}$ be the contraction $\left({ }^{8}\right)$ of $H$ with domain $\mathscr{D}_{1}$, and let $\widetilde{H}_{1}$ be its closure $\left({ }^{9}\right)$. We shall show that $\widetilde{H}_{1}$ is self-adjoint. Since $H$ is symmetric and $H \supseteq H_{1}\left({ }^{10}\right)$, it follows then that $\widetilde{H}=\widetilde{H}_{1}$ and $H$ is essentially self-adjoint, $\widetilde{H}$ being its unique self-adjoint extension.

The core of the present paper is the proof that $\tilde{H}_{1}$ is self-adjoint $(\$ \$ 1-6)$. For this purpose, we show first that the kinetic energy part $T_{1}$, of $H_{1}$, which is merely a Laplace operator in $3 s$-dimensional space, is essentially self-adjoint

(8) $H_{1}$ is a contraction of $H$ if $H$ is an extension of $H_{1}$.

$\left({ }^{9}\right) \sim$ is used to denote closure of an operator. (S), p. 45 .

(10) $H \supseteq H_{1}$ means that $H$ is an extension of $H_{1}$. 
(Lemma 1), and study the properties of the domain $\mathcal{D}_{0}$ of the self-adjoint operator $T_{0}=\widetilde{T}_{1}$ (Lemmas 2,3). Next we show that the potential energy $V$ is not only defined everywhere in $\mathcal{D}_{0}$, but it is also "infinitely small" compared with $T_{0}$ (Lemma 4), which is the result of assumptions (4) and (5). It is then easy to show that $T_{0}+V$, defined on $\mathscr{D}_{0}$, is self-adjoint (Lemma 5) and this is shown to be identical with $\widetilde{H}_{1}$ (Theorem 1 ).

The unique self-adjoint extension $\widetilde{H}=\widetilde{H}_{1}=T_{0}+V$ of $H$ is to be regarded as the correct Hamiltonian of our system. There remains no ambiguity about its domain $\mathcal{D}_{0}$ any longer. We can even state explicitly a necessary and sufficient condition that a given function belong to that domain. Thus the question of boundary conditions can be regarded as completely solved, at least theoretically. A remarkable fact is that the domain $\mathcal{D}_{0}$ of $\widetilde{H}$ is identical with that of $T_{0}$ and independent of the potential energy $V(\S 9)$.

Although the closed Hamiltonian $\widetilde{H}$ is no longer a differential operator in the elementary sense, it preserves many properties of the latter. In particular, the eigenfunctions of $\widetilde{H}$ are sufficiently differentiable and are solutions of the wave equation $\left({ }^{11}\right)(\S 8)$.

The remaining part of the paper is devoted to brief discussions on related topics such as the stability of Coulomb potentials against cut-off procedure, the variational method, and the perturbation method applied to our problem, of which a more detailed treatment will be given elsewhere.

1. Momentum space. Beside wave functions $f(\boldsymbol{r}) \equiv f\left(\boldsymbol{r}_{1}, \cdots, \boldsymbol{r}_{\boldsymbol{s}}\right)$ defined in the configuration space, we need their representation $F(\boldsymbol{p}) \equiv F\left(\boldsymbol{p}_{1}, \cdots, \boldsymbol{p}_{\boldsymbol{s}}\right)$ in the momentum space. Mathematically $F(\boldsymbol{p})$ are the Plancherel transforms $\left({ }^{12}\right)$ of $f(\boldsymbol{r})$. The correspondence $f(\boldsymbol{r}) \rightleftarrows F(\boldsymbol{p})$ is one-to-one, linear and isometric in the sense that

$$
\begin{aligned}
\|f\|^{2} \equiv \int|f(\boldsymbol{r})|^{2} d \boldsymbol{r}=\int|F(\boldsymbol{p})|^{2} d \boldsymbol{p} \equiv\|F\|^{2}, \\
\\
\left(d \boldsymbol{r} \equiv d \boldsymbol{r}_{1} \cdots d \boldsymbol{r}_{\boldsymbol{s}}, d \boldsymbol{p} \equiv d \boldsymbol{p}_{1} \cdots d \boldsymbol{p}_{\boldsymbol{s}}\right) .
\end{aligned}
$$

If $\int|f(\boldsymbol{r})| d \boldsymbol{r}$ exists, we have $\left({ }^{13}\right)$

$$
F(\boldsymbol{p})=(2 \pi)^{-3 s / 2} \int f(\boldsymbol{r}) \exp (-i \boldsymbol{p r}) d \boldsymbol{r} \quad\left(\boldsymbol{p r} \equiv \boldsymbol{p}_{1} \boldsymbol{r}_{1}+\cdots+\boldsymbol{p}_{s} \boldsymbol{r}_{s}\right),
$$

(11) In order to prove the existence of solutions of the wave equation, it is therefore suffcient to show the existence of eigenfunctions of the closed Hamiltonian $\widetilde{H}$. This is quite important, for the complicated problem regarding the partial differential equation can be reduced to a much easier analysis of the spectrum of a self-adjoint operator. We shall carry it out in the case of the helium atom in the following paper. p. 173.

(12) See for example S. Bochner, Vorlesungen ïber Fouriersche Integrale, Leipzig, 1932,

${ }^{(13)}$ Strictly speaking, we should add the words "almost everywhere." But we shall omit such provisos so long as there is no fear of misunderstanding. 
and if $\int|F(p)| d p$ exists, we have

$$
f(\boldsymbol{r})=(2 \pi)^{-38 / 2} \int F(\boldsymbol{p}) \exp (i \boldsymbol{p} \boldsymbol{r}) d \boldsymbol{p} .
$$

As is well known, the operator $i^{-1} \operatorname{grad}_{j}$ in the configuration space is formally represented by $\boldsymbol{p}_{j} \times$ in the momentum space.

It is convenient to view $f(\boldsymbol{r})$ and $F(\boldsymbol{p})$ as two representations of one and the same vector of abstract Hilbert space $\mathfrak{S}$. We can freely pass from one to the other. For instance, if we have defined an operator $R$ in the momentum space, it is also defined in the configuration space by virtue of the correspondence $f \rightleftarrows F$ and $R f \rightleftarrows R F$.

2. Kinetic energy. The kinetic energy of our system is formally given by

$$
T=-\sum_{i=1}^{s} \mu_{i} \operatorname{grad}_{i}^{2}-\mu_{0}\left(\sum_{i=1}^{s} \operatorname{grad}_{i}\right)^{2} .
$$

Now we define a multiplicative operator $T_{0}$ in the momentum space by $\left(T_{0} F\right)(p)=T_{0}(p) F(p)$, where

$$
T_{0}(\boldsymbol{p})=\sum_{i=1}^{s} \mu_{i} p_{i}^{2}+\mu_{0}\left(\sum_{i=1}^{8} p_{i}\right)^{2} .
$$

The domain $\mathcal{D}_{0}$ of $T_{0}$ is by definition the set of all functions $F(p) \in \mathfrak{S}$ such that $T_{0}(p) F(p) \in \mathfrak{S}$. Thus defined, $T_{0}$ is obviously self-adjoint $\left({ }^{14}\right)$. It is easily seen that $F(\boldsymbol{p})$ is in $\mathcal{D}_{0}$ if and only if it satisfies simultaneously

$$
\int|F(p)|^{2} d p<\infty, \quad \int p_{i}^{4}|F(p)|^{2} d p<\infty \quad(i=1, \cdots, s) .
$$

Consequently $\mathcal{D}_{0}$ does not depend on the constants $\mu_{0}, \mu_{1}, \cdots, \mu_{s}$ as long as $\mu_{i}>0$ for $i \geqq 1$. As we shall see, $T_{0}$ is the correct kinetic energy operator and coincides with $\tilde{T}$.

3. Function space $\mathcal{D}_{1}$. We have defined by (3) the set $\mathcal{D}_{1}$ of functions in configuration space. But it is well known that the Plancherel transform of a function (3) has a similar form with arguments $\boldsymbol{r}$ replaced by $\boldsymbol{p}$. Thus $\mathcal{D}_{1}$ is, represented in momentum space, the set of all functions of the form

$$
G(p)=P\left(p_{1}, \cdots, p_{s}\right) \exp \left\{-(1 / 2)\left(p_{1}^{2}+\cdots+p_{s}^{2}\right)\right\},
$$

where $P$ is a polynomial of its $3 s$ coordinates. Evidently (7) and (8) are true if $f(\boldsymbol{r})$ or $F(\boldsymbol{p})$ is in $\mathcal{D}_{1}$. Moreover, since $F(\boldsymbol{p}) \in \mathcal{D}_{1}$ vanishes at infinity exponentially, we can differentiate (8) under the integral sign as often as we

(14) See for example (S), p. 229. 
like. Hence $\mathscr{D}_{1} \subseteq \mathcal{D}_{0}$, and for $f \in \mathscr{D}_{1}, T_{0}$ coincides with the differential operator (9). Let $T_{1}$ be the contraction of $T$ with domain $\mathcal{D}_{1}$. Then we have the following lemma.

Lemma 1. $\tilde{T}_{1}=T_{0}$ and hence $T_{1}$ is essentially self-adjoint.

REMARK. Although the domain of $T$ was not specified, it follows that $\widetilde{T}=\widetilde{T}_{1}=T_{0}$ if we assume the trivial conditions (i), (ii) of the introduction for $T$ itself. Thus $T_{0}$ is the correct (self-adjoint) kinetic energy operator.

Proof. First we notice that $\mathcal{D}_{1}$ is a linear set dense in $\mathfrak{S}$, for $\mathcal{D}_{1}$ contains the Hermite orthogonal system which is known to be complete. Next we show that the set $\left(1+T_{0}\right) \mathcal{D}_{1}$ is also dense in $\mathfrak{S}$. For this purpose, it suffices to show that no $F_{0}(\boldsymbol{p}) \neq 0$ of $\mathfrak{S}$ can be orthogonal to all functions of $\left(1+T_{0}\right) \mathscr{D}_{1}$. Now $\left(\left(1+T_{0}\right) G, F_{0}\right)=0\left(G \in \mathcal{D}_{1}\right)$ means

$$
\int \overline{F_{0}(\boldsymbol{p})}\left(1+T_{0}(\boldsymbol{p})\right) P(\boldsymbol{p}) \exp \left\{-(1 / 2) \boldsymbol{p}^{2}\right\} d \boldsymbol{p}=0,
$$

where we have written $p^{2} \equiv p_{1}^{2}+\cdots+p_{s}^{2}$. The function $F_{0}(p)\left(1+T_{0}(p)\right)$ may not be in $\mathfrak{S}$, but $F_{0}(\boldsymbol{p})\left(1+T_{0}(\boldsymbol{p})\right) \exp \left\{-(1 / 4) \boldsymbol{p}^{2}\right\}$ is certainly in $\mathfrak{S}$, and this is orthogonal to all functions $P(p) \exp \left\{-(1 / 4) p^{2}\right\}$. But these are dense in $\mathfrak{S}$ too, for the proof given above is equally valid for the factor $1 / 2$ or $1 / 4$ in the exponential function. Thus

$$
F_{0}(\boldsymbol{p})\left(1+T_{0}(\boldsymbol{p})\right) \exp \left\{-(1 / 4) \boldsymbol{p}^{2}\right\}=0
$$

and, since $1+T_{0}(p)$ vanishes nowhere, $F_{0}$ must be zero.

Now if a $F(\boldsymbol{p}) \in \mathcal{D}_{0}$ is given, we have by the definition of $\mathcal{D}_{0},\left(1+T_{0}\right) F \in \mathfrak{S}$. As $\left(1+T_{0}\right) \mathcal{D}_{1}$ was shown to be dense in $\mathfrak{S}$, there exists to every $\epsilon>0$ a $G(p)$ $\in \mathcal{D}_{1}$ such that

$$
\left\|\left(1+T_{0}\right) G-\left(1+T_{0}\right) F\right\|<\epsilon,
$$

and hence by $T_{0}(p) \geqq 0$ we have

$$
\|G-F\|<\epsilon, \quad\left\|T_{0} G-T_{0} F\right\|<\epsilon,
$$

which means( $\left.{ }^{15}\right) \widetilde{T}_{1} \supseteq T_{0}$. But since $T_{0}$ is self-adjoint, it follows that $\widetilde{T}_{1}=T_{0}$.

4. Function space $\mathscr{D}_{0}$. A function of the domain $\mathscr{D}_{0}$ of the correct kinetic energy operator $T_{0}$ is characterized by (11) in momentum space. In configuration space, its characterization is also possible though not so simple. Here we shall not undertake to state the exact theorem and merely suggest that, roughly speaking, a function $f(\boldsymbol{r}) \in \mathfrak{S}$ is in $\mathcal{D}_{0}$ if and only if it has almost everywhere derivatives of the second order which are functions of $\mathfrak{S}$. A function of $\mathcal{D}_{0}$ is thus in general not differentiable in the usual sense. It can even be

(15) This is just the definition of closure. (S), p. 45. 
shown that if $s \geqq 2$, there exist functions of $\mathscr{D}_{0}$ which are everywhere discontinuous essentially $\left({ }^{16}\right)$. We have, however, the following lemma.

LemmA 2. If $s=1$, all functions $f(\boldsymbol{r}) \in \mathcal{D}_{0}$ are bounded and uniformly continuous $\left({ }^{17}\right)$.

Proof. For $f \rightleftarrows F$ we have

$$
\int|F(p)| d p \leqq\left\{\int|F(p)|^{2}\left(1+p^{4}\right) d p \int\left(1+p^{4}\right)^{-1} d p\right\}^{1 / 2}<\infty
$$

by the Schwarz inequality and (11). Hence by (8) we have

$$
f(\boldsymbol{r})=(2 \pi)^{-3 / 2} \int F(\boldsymbol{p}) \exp (i \boldsymbol{p r}) d \boldsymbol{p},
$$

and the assertion follows easily.

The lemma can partly be generalized to the case $s \geqq 2$ as follows. Define for every $f(\boldsymbol{r}) \in \mathfrak{G}$ and $i=1, \cdots, s$

$$
A_{i} f\left(\boldsymbol{r}_{i}\right)=\left\{\int|f(\boldsymbol{r})|^{2} d \boldsymbol{r}_{1} \cdots d \boldsymbol{r}_{i-1} d \boldsymbol{r}_{i+1} \cdots d \mathbf{r}_{s}\right\}^{1 / 2} .
$$

$A_{i}$ is an operator whose domain is $\mathfrak{E}$ and whose range is in the Hilbert space $\mathfrak{E}_{i}=L_{2}\left(\boldsymbol{r}_{i}\right)$ defined over the whole 3-dimensional space $\left(\boldsymbol{r}_{i}\right) . A_{i}$ is not linear, but we can easily show that

$$
\begin{aligned}
\left\|A_{i} f\right\| & =\|f\|, \\
\left|\left(A_{i} f-A_{i} g\right)\left(\boldsymbol{r}_{i}\right)\right| & \leqq A_{i}(f-g)\left(\boldsymbol{r}_{i}\right) \quad(i=1, \cdots, s), \\
\left\|A_{i} f-A_{i} g\right\| & \leqq\|f-g\| .
\end{aligned}
$$

Lemma 3. If $f(\boldsymbol{r}) \in \mathcal{D}_{0}$, then $A_{i} f(i=1, \cdots, s)$ are continuous and bounded as

$$
0 \leqq A_{i} f\left(\boldsymbol{r}_{i}\right) \leqq a^{\prime}\left\|T_{0} f\right\|+b^{\prime}\|f\|,
$$

where $a^{\prime}, b^{\prime}$ are constants independent of $f$, and $a^{\prime}$ can be taken as small as we like.

Proof. It is sufficient to prove the lemma for $i=1$. First let $g$ be a function of $\mathcal{D}_{1}$. Then we have by $(8)$

$$
\begin{aligned}
g(\boldsymbol{r})= & (2 \pi)^{-3 s / 2} \int \exp \left\{i\left(\boldsymbol{p}_{2} \boldsymbol{r}_{2}+\cdots+\boldsymbol{p}_{s} \boldsymbol{r}_{s}\right)\right\} d p_{2} \cdots d p_{s} \\
& \cdot \int \exp \left(i \boldsymbol{p}_{1} \boldsymbol{r}_{1}\right) G\left(\boldsymbol{p}_{1}, \boldsymbol{p}_{2}, \cdots, \boldsymbol{p}_{s}\right) d \boldsymbol{p}_{1},
\end{aligned}
$$

${ }^{(16)}$ That is, the discontinuity cannot be eliminated by changing the values of functions at points of a null set.

(17) It implies that the wave functions for stationary states of hydrogen-like atoms are bounded and continuous. See $\$$. 
since the order of integration is arbitrary on account of rapid decrease at infinity of $G(p)$. The Parseval identity applied to $3(s-1)$ variables $\boldsymbol{r}_{2}, \cdots, \boldsymbol{r}_{s}$ then gives

$$
\begin{aligned}
\left|A_{1} g\left(\boldsymbol{r}_{1}\right)\right|^{2} & =\int|g(\boldsymbol{r})|^{2} d \mathbf{r}_{2} \cdots d \boldsymbol{r}_{s} \\
& =(2 \pi)^{-3} \int d \boldsymbol{p}_{2} \cdots d \boldsymbol{p}_{\boldsymbol{s}}\left|\int \exp \left(i \boldsymbol{p}_{1} \boldsymbol{r}_{1}\right) G(\boldsymbol{p}) d \boldsymbol{p}_{1}\right|^{2} \\
& \leqq(2 \pi)^{-3} \int d \boldsymbol{p}_{2} \cdots d \boldsymbol{p}_{s}\left\{\int|G(\boldsymbol{p})| d \boldsymbol{p}_{1}\right\}^{2} .
\end{aligned}
$$

But we have as in the proof of Lemma 2

$$
\left\{\int|G(p)| d p_{1}\right\}^{2} \leqq \int|G(p)|^{2}\left(1+k^{4} p_{1}^{4}\right) d p_{1} \cdot \int\left(1+k^{4} p_{1}^{4}\right)^{-1} d p_{1}
$$

where $k$ is an arbitrary positive constant, all integrals converging absolutely. The last factor is easily seen to be

$$
\int\left(1+k^{4} p_{1}^{4}\right)^{-1} d p_{1}=c k^{-3},
$$

where $c$ is a numerical constant. Putting (18) into (17) and integrating with respect to $p_{2}, \cdots, p_{s}$, we have by (16)

$$
\begin{aligned}
\left|A_{1} g\left(\boldsymbol{r}_{1}\right)\right|^{2} & \leqq(2 \pi)^{-3} c k^{-3} \int|G(\boldsymbol{p})|^{2}\left(1+k^{4} \boldsymbol{p}_{1}^{4}\right) d p_{1} \cdots d p_{s} \\
& =(2 \pi)^{-3} c k^{-3}\left(\|G\|^{2}+k^{4}\left\|\boldsymbol{p}_{1}^{2} G\right\|^{2}\right) .
\end{aligned}
$$

But as $\left\|T_{0} G\right\| \geqq \mu_{1}\left\|p_{1}^{2} G\right\|$, we have by $g \rightleftarrows G$

$$
\left|A_{1} g\left(\boldsymbol{r}_{1}\right)\right|^{2} \leqq(2 \pi)^{-3} c\left(k \mu_{1}^{-2}\left\|T_{0} g\right\|^{2}+k^{-3}\|g\|^{2}\right) .
$$

Hence there are constants $a^{\prime}, b^{\prime}$ such that

$$
\begin{aligned}
0 & \leqq A_{1} g\left(\boldsymbol{r}_{1}\right) \\
& \leqq a^{\prime}\left\|T_{0} g\right\|+b^{\prime}\|g\|
\end{aligned}
$$

and since $k$ was arbitrary, $a^{\prime}$ can be made as small as we like by taking $k$ sufficiently small.

We have shown (19) only for $g \in \mathcal{D}_{1}$, but it can easily be extended to all $f \in \mathcal{D}_{0}$. According to Lemma 1 , we can take for every $f \in \mathcal{D}_{0}$ a sequence $\left\{g_{n}\right\}$ from $\mathcal{D}_{1}$ such that

$$
\left\|g_{n}-f\right\| \rightarrow 0, \quad\left\|T_{0} g_{n}-T_{0} f\right\| \rightarrow 0 \quad(n \rightarrow \infty) .
$$


Then we have by (14) and (19)

$$
\begin{aligned}
\left|A_{1} g_{m}\left(\boldsymbol{r}_{1}\right)-A_{1} g_{n}\left(\boldsymbol{r}_{1}\right)\right| & \leqq A_{1}\left(g_{m}-g_{n}\right)\left(\boldsymbol{r}_{1}\right) \\
& \leqq a^{\prime}\left\|T_{0} g_{m}-T_{0} g_{n}\right\|+b^{\prime}\left\|g_{m}-g_{n}\right\| \rightarrow 0
\end{aligned}
$$

when $m, n \rightarrow \infty$. The sequence $\left\{A_{1} g_{n}\left(\boldsymbol{r}_{1}\right)\right\}$ is therefore uniformly convergent. On the other hand we have by (14)

$$
\left\|A_{1} g_{n}-A_{1} f\right\| \leqq\left\|g_{n}-f\right\| \rightarrow 0 \quad(n \rightarrow \infty),
$$

and $\left\{A_{1} g_{n}\right\}$ converges in the mean to $A_{1} f$. It follows that a suitable subsequence of $\left\{A_{1} g_{n}\right\}$ converges uniformly to $A_{1} f$ almost everywhere, and if we make $n \rightarrow \infty$ appropriately in

$$
\begin{aligned}
0 & \leqq A_{1} g_{n}\left(\boldsymbol{r}_{1}\right) \\
& \leqq a^{\prime}\left\|T_{0} g_{n}\right\|+b^{\prime}\left\|g_{n}\right\|,
\end{aligned}
$$

we obtain (15) for $i=1$. Since $A_{1} g_{n}$ are all continuous, the limit function $A_{1} f$ is also continuous.

5. Potential energy. Next we consider the potential energy $V$. The meaning of the operator $V$ is clear: it is a real multiplicative operator, and its domain $\mathscr{D}_{V}$ consists of all $f \in \mathfrak{S}$ for which $V f \in \mathfrak{S}$. Thus defined, $V$ is obviously self-adjoint $\left({ }^{14}\right)$.

Lemma 4. The domain $\mathcal{D}_{V}$ of $V$ contains $\mathcal{D}_{0}$ and there are two constants $a, b$ such that

$$
\|V f\| \leqq a\left\|T_{0} f\right\|+b\|f\|
$$

for every $f \in \mathcal{D}_{0}$. Moreover, a can be taken as small as we like.

Proof. It suffices to show this for the cases $V=V^{\prime}\left(\boldsymbol{r}_{i}\right), V=V_{0 i}(\boldsymbol{r})$ and $V=V_{i j}\left(\boldsymbol{r}_{i}-\boldsymbol{r}_{j}\right)$ separately (see (4)). The case $V=V^{\prime}$ is trivial, for, as $V^{\prime}$ is bounded by (5), we can take $a=0, b=C$. Next we consider the case $V=V_{01}\left(\boldsymbol{r}_{1}\right)$. We have by (13)

$$
\begin{aligned}
\left\|V_{01} f\right\|^{2} & =\int\left|V_{01}\left(\boldsymbol{r}_{1}\right)\right|^{2}\left|f\left(\boldsymbol{r}_{1}, \cdots, \boldsymbol{r}_{s}\right)\right|^{2} d \boldsymbol{r}_{1} \cdots d \boldsymbol{r}_{s} \\
& =\int\left|V_{01}\left(\boldsymbol{r}_{1}\right)\right|^{2} \cdot\left|A_{1} f\left(\boldsymbol{r}_{1}\right)\right|^{2} d \boldsymbol{r}_{1} \\
& =\int_{r_{1} \leqq R}+\int_{\boldsymbol{r}_{1}>R} \cdot
\end{aligned}
$$

If $f \in \mathcal{D}_{0}$, we have by Lemma 3

$$
\left|A_{1} f\left(\boldsymbol{r}_{1}\right)\right|^{2} \leqq 2 a^{\prime 2}\left\|T_{0} f\right\|^{2}+2 b^{\prime 2}\|f\|^{2},
$$


and hence by (21), (5), and (14)

$$
\begin{aligned}
\left\|V_{01} f\right\|^{2} \leqq & \left(2 a^{\prime 2}\left\|T_{0} f\right\|^{2}+2 b^{\prime 2}\|f\|^{2}\right) \int_{r_{1} \leqq R}\left|V_{01}\left(\boldsymbol{r}_{1}\right)\right|^{2} d \boldsymbol{r}_{1} \\
& +C^{2} \int_{r_{1}>R}\left|A_{1} f\left(\boldsymbol{r}_{1}\right)\right|^{2} d \boldsymbol{r}_{1} \\
\leqq & C^{2}\left(2 a^{\prime 2}\left\|T_{0} f\right\|^{2}+2 b^{\prime 2}\|f\|^{2}\right)+C^{2}\|f\|^{2} .
\end{aligned}
$$

Since $a^{\prime}$ can be chosen as small as we like, we obtain (20) for $V=V_{01}\left(\boldsymbol{r}_{1}\right)$.

In the case $V=V_{12}\left(\boldsymbol{r}_{1}-\boldsymbol{r}_{2}\right)$, change variables according to

$$
\begin{gathered}
r_{1}^{\prime}=r_{1}-r_{2}, r_{2}^{\prime}=r_{2}, \cdots, r_{s}^{\prime}=r_{s}, \\
d r_{1} \cdots d r_{s}=d r_{1}^{\prime} \cdots d r_{s}^{\prime}, \\
f\left(r_{1}, \cdots, r_{8}\right)=f^{\prime}\left(r_{1}^{\prime}, \cdots, r_{s}^{\prime}\right) .
\end{gathered}
$$

Then we have

$$
\left\|V_{12} f\right\|^{2}=\int\left|V_{12}\left(\boldsymbol{r}_{1}^{\prime}\right)\right|^{2} \cdot\left|f^{\prime}\left(\boldsymbol{r}_{1}^{\prime}, \cdots, \boldsymbol{r}_{s}^{\prime}\right)\right|^{2} d \mathbf{r}_{1}^{\prime} \cdots d \mathbf{r}_{8}^{\prime}
$$

But it can easily be shown that $f \in \mathcal{D}_{0}$ implies $f^{\prime} \in \mathcal{D}_{0}$ (we have only to consider the $p$-representation of $\left.f^{\prime}\right)$, so that (20) follows for $V=V_{12}\left(\boldsymbol{r}_{1}-\boldsymbol{r}_{2}\right)$ in the same way as above.

\section{Proof of the main theorem.}

Lemma 5. The operator $T_{0}+V$ defined on $\mathcal{D}_{0}$ is self-adjoint.

Proof. $\left({ }^{18}\right)$. It is sufficient to show that the range of $T_{0}+V \pm i k$ coincides with $\mathfrak{S}$ for some $k>0$. Since $T_{0}$ is self-adjoint, $\left(T_{0} \pm i k\right)^{-1}$ is a linear operator defined everywhere in $\mathfrak{S}$ and its range is $\mathscr{D}_{0}$. Hence we have by (20) for every $\phi \in \mathfrak{S}$

$$
\begin{aligned}
\left\|V\left(T_{0} \pm i k\right)^{-1} \phi\right\| & \left.\leqq a \| T_{0}\left(T_{0} \pm i k\right)^{-1}\right) \phi\|+b\|\left(T_{0} \pm i k\right)^{-1} \phi \| \\
& \leqq a\|\phi\|+b k^{-1}\|\phi\| .
\end{aligned}
$$

According to Lemma 4 we can assume $a<1$. Then, if we take $k$ sufficiently large, we have $a+b k^{-1}<1$. Thus the operator $V\left(T_{0} \pm i k\right)^{-1}$ is defined everywhere in $\mathfrak{S}$ and its bound is less than unity. Consequently the range of $1+V\left(T_{0} \pm i k\right)^{-1}$ is the whole space $\mathfrak{S}\left({ }^{19}\right)$. From

$$
T_{0}+V \pm i k=\left\{1+V\left(T_{0} \pm i k\right)^{-1}\right\}\left(T_{0} \pm i k\right),
$$

(18) As regards the theorems concerning self-adjoint operators used here, see (S), chap. IV.

(19) If a bounded linear operator $A$ has bound less than unity, $(1+A)^{-1}=1-A+A^{2}-\cdots$ exists and is defined everywhere. Hence the range of $1+A$ is $\mathfrak{\$}$. 
it follows that the range of $T_{0}+V \pm i k$ is $\mathfrak{S}$, for the range of $T_{0} \pm i k$ is also $\mathfrak{S}$ since $T_{0}$ is self-adjoint.

REMARK. We note without proof that the operator $T_{0}+V$ is bounded below. In any case, this is easily seen if $V$ is of Coulomb type [(K), p. 207].

THEOREM 1. $H_{1}$ is essentially self-adjoint, and its unique self-adjoint extension $\widetilde{H}_{1}$ coincides with $T_{0}+V$.

Proof. $H_{1}$ was defined in the introduction. Its domain is $\mathscr{D}_{1}$ and it is clear that $H_{1}=T_{1}+V \subseteq T_{0}+V$. Since $T_{0}+V$ was shown to be self-adjoint, we have only to show that to every $f \in \mathscr{D}_{0}$ and $\epsilon>0$, there exists a $g \in \mathscr{D}_{1}$ such that

$$
\|g-f\|<\epsilon, \quad\left\|\left(T_{0}+V\right)(g-f)\right\|<\epsilon .
$$

But the existence of such a $g$ is concluded easily from Lemmas 1 and 4.

7. Remarks and supplements. We have shown that $H_{1}$ is essentially selfadjoint. It follows from the reasoning of the introduction that the given operator $H$ is also essentially self-adjoint, $\widetilde{H}=\widetilde{H}_{1}=T_{0}+V$ being its unique self-adjoint extension. Since all operators representing physical quantities are required to be self-adjoint $\left({ }^{1}\right)\left({ }^{2}\right)$, we can conclude without ambiguity that $\widetilde{H}=T_{0}+V$ is the correct Hamiltonian of our system. It is important to notice that this correct Hamiltonian is uniquely determined by assumptions (i), (ii) of the introduction, which are almost trivial and will certainly be admitted by everyone, although there may be differences of opinions about the precise domain of the initial operator $H$. But this difference of opinions is irrelevant, for $\widetilde{H}$, and not $H$, is the correct Hamiltonian of our system and this is defined precisely as $T_{0}+V$ with domain $\mathscr{D}_{0}$.

That $\widetilde{H}$ is self-adjoint means that $\widetilde{H}$ has a complete (discrete or continuous) set of eigenfunctions, but our result gives us no information about the nature of its spectrum, except that it is bounded below and unbounded above. In particular, we cannot conclude the existence of discrete eigenvalues disclosed in experiment. It will require more detailed calculation before we can derive the fact theoretically. The writer wishes to treat in the following paper the problem in the case of the helium atom and show there the existence of a large number of eigenvalues.

The above theory does not comprise such problems as the Zeeman and Stark effects. The Hamiltonian of the Zeeman effect for our system can be shown to be essentially self-adjoint by a modification of the above method if we assume Coulomb interaction between particles. In the case of the Stark effect, however, we have hitherto succeeded in obtaining the same result only in the simplest case of the hydrogen-like atom. The difficulty arises from the fact that the potential of the external electric field is not bounded below in a large part of configuration space.

Our method is also applicable to the Dirac relativistic Hamiltonian of the hydrogen-like atom and gives similar results unless the atomic number $Z$ is 
too large $(Z \leqq 68)\left({ }^{20}\right)$. The chief difference from the nonrelativistic case is that the domain of the correct Hamiltonian is characterized here by

$$
\int\left|F_{k}(\boldsymbol{p})\right|^{2} d \boldsymbol{p}<\infty, \quad \int \boldsymbol{p}^{2}\left|F_{k}(\boldsymbol{p})\right|^{2} d \boldsymbol{p}<\infty \quad(k=1,2,3,4)
$$

where $F_{k}(p)$ are four components of the wave function represented in momentum space, whereas $\mathcal{D}_{0}$ was characterized by (11) in the nonrelativistic case.

8. Differentiability of eigenfunctions. An important question is whether the eigenfunctions for discrete eigenvalues of $\tilde{H}$, if they exist, are really solutions of the wave equation considered as a usual differential equation. They are indeed solutions of the operational equation $\widetilde{H} \phi=\lambda \phi$, but $\widetilde{H}$ is not necessarily a differential operator in the usual sense (see $\$ 4$ ). Of course they are in the domain $\mathcal{D}_{0}$ of $\tilde{H}$, but a function of $\mathcal{D}_{0}$ is not necessarily differentiable in the ordinary sense (see $\S 4$ ). Thus the subject requires a particular investigation.

Fortunately we have at our disposal a group of theorems concerning general differential operators of elliptic type derived by several mathematicians $\left({ }^{21}\right)$. Here we shall resort to Friedrichs's theorem. Before applying his theorem $\left({ }^{22}\right)$ to our problem, however, we must take into account that our potential $V(\boldsymbol{r})$ has singular points and that, moreover, his operator $D^{*} D$ is not necessarily identical with our kinetic energy $T_{0}$, even if we make both coincide formally by a linear transformation of coordinates, which is assumed to be done in the following. But since Friedrichs's theory is essentially of a local character, the first difficulty can be avoided by taking as the ground region $S$ a small portion of configuration space where the potential $V(\boldsymbol{r})$ is sufficiently regular. Then we inquire into the relation between his operator $D^{*} D$ considered on $S$ and our $T_{0}$. We can show without difficulty that, if $f(\boldsymbol{r}) \in \mathcal{D}_{0}$, then $D^{*} D f$ exists when we consider $f$ as a function defined on $S$ and

${ }^{(20)}$ The figure 68 seems to result from our method and to have no serious meaning. In any case, however, some restriction on $Z$ is necessary in order that the Dirac operator be essentially self-adjoint. In a paper to appear in the Physical Review, K. M. Case discusses the solutions of the Dirac equation in greater detail by the method of series expansion and, in particular, shows that a new boundary condition must be introduced at the force-center if $Z>137$. Actually it seems to the writer that perhaps the Dirac operator can be extended, in a natural and unique manner, to a self-adjoint operator provided $Z<137$. But the domain of the latter is no longer characterized by (22), and perhaps depends on $Z$, at least if $Z>119$. Thus the problem is rather complicated in contrast with the Schrödinger operator. The writer wishes to thank Professor J. von Neumann and Dr. K. M. Case who kindly afforded facilities for seeing the paper before publication.

$\left.{ }^{21}\right)$ K. O. Friedrichs, Amer. J. Math. vol. 61 (1939) p. 523; H. Weyl, Duke Math. J. vol. 7 (1940) p. 411; K. Kodaira, Proc. Imp. Acad. Tokyo vol. 20 (1944) p. 262.

(22) K. Friedrichs, footnote 21, Theorem 15.3. 
there coincides with the function $T_{0} f$. Now let $\phi$ be an eigenfunction of $\tilde{H}$ : $\widetilde{H} \phi=\left(T_{0}+V\right) \phi=\lambda \phi$. By the result just stated we have $D^{*} D \phi+(V-\lambda) \phi=0$ in $S$. Thus Freidrichs's theorem is applicable and we derive the following theorem.

TheOREM 2. An eigenfunction $\phi$ of $\tilde{H}$ has continuous derivatives of the second order in a region $S$ of configuration space where the potential $V(\boldsymbol{r})$ is sufficiently regular $\left({ }^{23}\right)$, and satisfies the wave equation.

If $V$ is the Coulomb potential as in the case of real atoms, it follows that the eigenfunctions satisfy the wave equation everywhere except at singular points of the potential $\left({ }^{24}\right)$. Regarding their behavior at these singular points, we can derive no conclusion from the above theorem. A detailed study shows, however, that they are bounded even at such points.

9. Boundary conditions. There are diverse opinions $\left({ }^{7}\right)$ about the boundary and continuity conditions to be imposed on solutions of the wave equation in order that they be eigenfunctions of the Hamiltonian operator. Boundary conditions are necessary simply because a differential operator becomes a self-adjoint operator of Hilbert space only if suitably chosen boundary conditions are added to it. In general, the resulting operator is different according to different choice of boundary conditions.

In our problem, we have shown that $H$ is essentially self-adjoint without any artificial boundary conditions $\left({ }^{25}\right)$. This means that the boundary conditions are of themselves determined uniquely. Since the correct Hamiltonian $\widetilde{H}=T_{0}+V$ is precisely defined with domain $\mathcal{D}_{0}$, there remains no ambiguity about them.

From the abstract point of view, we have no need of such things as boundary conditions, for a $\phi \in \mathfrak{S}$ is an eigenfunction of $\widetilde{H}$ if and only if $\tilde{H} \phi=\lambda \phi$ for a real number $\lambda$. For practical purposes, however, we are in many cases obliged to look at the wave equation as a differential equation and select the eigenfunctions of $\tilde{H}$ from among solutions of the wave equation $\left({ }^{26}\right)$. If a solution $\phi$ is found which satisfies the wave equation except possibly at singular points of the potential energy, it can be shown (under certain general conditions) that $\phi$ is an eigenfunction for a discrete eigenvalue of the Hamiltonian $\widetilde{H}$ if and only if $\phi$ lies in $\mathcal{D}_{0}$. Since $\phi \in \mathcal{D}_{0}$ is equivalent to (11), we can say that complete (that is, necessary and sufficient) boundary and continuity conditions are given by (11) in terms of momentum representation.

(23) According to Friedrichs's theorem, it is sufficient if $V(\boldsymbol{r})$ has continuous derivatives of the $([3 s / 2]+3)$ th order. But the figure is of no importance. We can show by an improved theory that it can be reduced to 1 .

(24) They are even analytic since the Coulomb potential is an analytic function.

(25) Such are of ten necessary when the system is enclosed in a "box."

(26) Since all eigenfunctions are shown to be solutions of the wave equation ( $\$ 8$ ), we have no fear of losing any one of the eigenfunctions in this way. 
They can also be stated directly in the configuration space as was suggested in $\S 4\left({ }^{27}\right)$.

We can derive other boundary conditions of simpler form which are, however, necessary but not sufficient or vice versa. An example is given by Lemma 2. It shows that the boundary condition originally introduced by Schrödinger was a correct one $\left({ }^{28}\right)$. Often we encounter the statement that a wave function must be of order $o\left(r^{-1}\right)$ at a singular point, but this is less precise than the Schrödinger condition. By the way, it will be noted that Lemma 2 is sufficient to exclude the "second solutions" of $S$-state for the hydrogen-like wave equation [cf. (K), p. 153].

It is remarkable that the domain $\mathscr{D}_{0}$ of the correct Hamiltonian $\widetilde{H}$ is identical with that of $T_{0}$, which is the correct kinetic energy operator of our system and which can also be interpreted as the Hamiltonian of free particles. Our potential $V$ does not affect the domain of the total Hamiltonian in the least. It means, in particular, that the Coulomb potential is rather a mild one in spite of its singularity at force-centers $\left({ }^{29}\right)$. Singular points of the potential are in this respect not distinguished from other points. This is rather striking in view of apparently contrary statements in current literature. But it should be noticed that this property of $\mathscr{D}_{0}$ does not contradict the fact that eigenfunctions of $\widetilde{H}$ are in general singular only at singular points of $V(\boldsymbol{r})$, for eigenfunctions are as such far more regular $\left({ }^{24}\right)$ at regular points of $V(\boldsymbol{r})$ than general functions of $\mathscr{D}_{0}$.

The domain of the Dirac Hamiltonian is characterized by (22), which is much weaker than the corresponding condition (11) for the Schrödinger Hamiltonian, for $\boldsymbol{p}^{4}$ in the latter is replaced by $\boldsymbol{p}^{2}$. In consequence, the oomponents $f_{k}(\boldsymbol{r})$ of the wave function in the domain of the Dirac Hamiltonian are not necessarily bounded in contrast with the nonrelativistic case for $s=1$. We can see in this a characteristic feature of the Dirac Hamiltonian, which is well known to have nonbounded eigenfunctions.

10. Relation to the variational method. In the following we assume that

$\left.{ }^{27}\right)$ We have another indirect, but of ten convenient, criterion: $f(\boldsymbol{r}) \in \mathfrak{W}$ is in $\mathcal{D}_{0}$ if and only if there is a $f^{\prime}(r) \in \mathfrak{W}$ such that $(f, T g)=\left(f^{\prime}, g\right)$ for every $g \in \mathcal{D}_{1}$, that is, for every $g$ of the form (3). This is an immediate consequence of the fact that $T_{1}$ is essentially self-adjoint. See (S), chap. II.

${ }^{(28)}$ If $s \geqq 2$, functions of $\mathcal{D}_{0}$ are in general not bounded (\$4). As we indicated in $\$ 8$, however, eigenfunctions of $\widetilde{H}$ are nevertheless bounded. Thus Schrödinger's boundary condition is correct even for $s \geqq 2$ if applied to eigenfunctions.

${ }^{(29)}$ Some doubts have been expressed about the validity of the Coulomb force from mathematical considerations, cf. (K), p. 211. But our result will be sufficient to remove these doubts, all the more when we notice that the existence of solutions of the helium wave equation can be shown $(\$ \$ 7,8)$. Of course there are physical reasons why the Coulomb law cannot be absolutely correct, but it is quite important that the eigenvalue problem is still completely solvable even if we adopt the Coulomb potential with its singularities (\$12). 
the lowest part of the spectrum of $\tilde{H}$ consists of discrete eigenvalues $\left({ }^{30}\right)$

$$
\lambda_{1} \leqq \lambda_{2} \leqq \cdots
$$

with corresponding normalized eigenfunctions $\phi_{1}, \phi_{2}, \cdots$ As is well known, the lowest eigenvalue $\lambda_{1}$ is characterized as the minimum value of the quadratic form $(H \phi, \phi)$ under the supplementary condition $\|\phi\|=1$, the corresponding $\phi$ being $\phi_{1}\left({ }^{31}\right)$.

We have shown in the proof of Theorem 1 (\$6) that to every $f \in \mathcal{D}_{0}$ and $\epsilon>0$, there exists a $g \in \mathcal{D}_{1}$ such that $\|g-f\|<\epsilon,\left\|\left(T_{0}+V\right)(g-f)\right\|<\epsilon$. Since $\phi_{1}$ is in $\mathcal{D}_{0}$ and $T_{0}+V=\widetilde{H}$, it follows that there is a sequence $\left\{g_{n}\right\}$ such that

$$
g_{n} \in \mathcal{D}_{1}, \quad\left\|g_{n}-\phi_{1}\right\| \rightarrow 0, \quad\left\|\tilde{H} g_{n}-\tilde{H} \phi_{1}\right\| \rightarrow 0 \quad(n \rightarrow \infty) .
$$

We may assume that $\left\|g_{n}\right\|=1$, for otherwise we can replace $g_{n}$ by $\left\|g_{n}\right\|^{-1} g_{n}$ by virtue of $\left\|g_{n}\right\| \rightarrow\left\|\phi_{1}\right\|=1$. Consequently we have

$$
\left\|g_{n}\right\|=1, \quad\left(\tilde{H} g_{n}, g_{n}\right) \rightarrow\left(\tilde{H} \phi_{1}, \phi_{1}\right)=\lambda_{1} \quad(n \rightarrow \infty) .
$$

This relation means that $\lambda_{1}$ is the lower limit of $(H \phi, \phi)$ for $\|\phi\|=1$ when we restrict $\phi$ to $\mathcal{D}_{1}$. It is easily seen that a similar relation holds with respect to higher eigenvalues, $\lambda_{2}, \lambda_{3}, \cdots$, taking into account further supplementary conditions $\left({ }^{32}\right)$. But $\mathscr{D}_{1}$ was the set of all finite linear combinations of Hermite orthogonal functions of $3 s$ variables $\boldsymbol{r}_{1}, \boldsymbol{r}_{2}, \cdots, \boldsymbol{r}_{\mathrm{s}}$. Thus we have the result: the Ritz variational method applied to the quadratic form

$$
\begin{aligned}
\int\left[\sum_{i=1}^{s} \mu_{i}\left\{\left|\frac{\partial \phi}{\partial x_{i}}\right|^{2}+\left|\frac{\partial \phi}{\partial y_{i}}\right|^{2}+\left|\frac{\partial \phi}{\partial z_{i}}\right|^{2}\right\}\right. \\
\left.+\mu_{0}\left\{\left|\sum_{i=1}^{s} \frac{\partial \phi}{\partial x_{i}}\right|^{2}+\left|\sum_{i=1}^{s} \frac{\partial \phi}{\partial y_{i}}\right|^{2}+\left|\sum_{i=1}^{s} \frac{\partial \phi}{\partial z_{i}}\right|^{2}\right\}+V|\phi|^{2}\right] d r,
\end{aligned}
$$

with Hermite orthogonal functions as the basis, converges to the correct solutions of the eigenvalue problem of the Hamiltonian $\widetilde{H}\left({ }^{33}\right)$.

It is obvious from our theory $(\$ \$ 1-6)$ that the advantage of Hermite functions just stated has its origin in the property that the set $\left(1+T_{0}\right) \mathcal{D}_{1}$ is dense in $\mathfrak{S}$ (see the proof of Lemma 1). Hence we have the following theorem.

(30) Degenerate eigenvalues are counted repeatedly.

(3i) $\phi_{1}$ is not uniquely determined.

(32) See for example (K), p. 409.

${ }^{(33)}$ In this connection we should mention the theory of K. Friedrichs, Math. Ann. vol. 109 (1934) p. 465, which can be regarded as a generalization of the variational method. It gives a general method of assigning in a unique way a self-adjoint extension to every half-bounded symmetric operator, even when the latter is not essentially self-adjoint and hence has an infinity of different self-adjoint extensions. In our case $H_{1}$ was essentially self-adjoint, so that its Friedrichs extension naturally coincides with its unique self-adjoint extension $\tilde{H}$. The abovestated property of Hermite functions follows from this immediately. 
Theorem 3. A sufficient condition that the Ritz variational method applied to the quadratic form (23) with a given (not necessarily orthogonal) system of functions $u_{1}, u_{2}, \cdots$ as the basis converge to correct solutions of the eigenvalue problem of our Hamiltonian is that the system of functions $v_{n}=\left(c+T_{0}\right) u_{n}$ $(n=1,2, \cdots)$ be complete, that is, that the set of all finite linear combinations of them be dense in $\mathfrak{S}$, where $c$ is an arbitrary (but fixed) positive constant (34).

It should be noted that the condition is stated in terms of the kinetic energy only, the troublesome potential energy being entirely eliminated $\left.{ }^{35}\right)$. But it seems to be impracticable unless we know explicitly the representation of $u_{n}$ in momentum space, which was the case for the Hermite functions. In fact, we do not know at present whether certain other familiar orthogonal systems of functions satisfy the condition.

11. Perturbation theory and one-particle model. It is usual in atom mechanics to regard the mutual interaction of electrons as a small correction to the approximate (unperturbed) Hamiltonian composed of the kinetic energy and the potential energy of each electron due to the nucleus and some mean field produced by other electrons. Thus we have a decomposition

$$
H(\kappa)=H^{(0)}+\kappa H^{(1)},
$$

where $H^{(0)}$ corresponds to a certain one-electron model and $\kappa H^{(1)}$ is regarded as a small perturbation, $\kappa$ being a parameter taking the value 1 for the real system and 0 for the unperturbed system. Then the perturbation theory is applied to (24).

This way of applying the perturbation method to the problem of atoms is not only useful in practical computations, but also theoretically quite important, for it provides us with a sound basis for assigning a definite set of quantum numbers to each energy level of the real system $\left({ }^{36}\right)$.

But a perfect justification of applying the perturbation method has hitherto been lacking. Indeed, a mathematical foundation of the perturbation method is rather difficult, although it is employed so often in quantum mechanics, and in many cases its formal series is not convergent. For this reason, it is quite striking that the perturbation method actually converges in our problem. This is due to the favorable behavior of our potential $V$ expressed in Lemma 4, and follows from a theorem derived by Rellich in his papers on the perturbation theory $\left({ }^{37}\right)$.

(34) A detailed study shows that the condition can be weakened by replacing $c+T_{0}$ by $\left(c+T_{0}\right)^{1 / 2}$, the latter being best defined in terms of momentum space.

${ }^{(35)}$ Of course under the assumptions (4) and (5).

(36) An exhaustive treatment of the subject is given in (K), pp. 484-491.

(37) F. Rellich, Math. Ann. vol. 113 (1937) pp. 600, 677; vol. 116 (1939) p. 555; vol. 117 (1940) p. 356. In particular, see Theorem 5 of his third paper. The writer of the present paper also developed a mathematical theory of perturbation by a different method. The result will be published elsewhere. An abridged account has been given in Progress of Theoretical Physics vol. 4 (1949) p. 514, vol. 5 (1950) pp. 95, 207. 
In fact, it can be shown that the (discrete) eigenvalues and eigenfunctions of $H(\kappa)$ are not only continuous, but analytic functions of $\kappa$, provided that the decomposition of the potential energy $V$ into two parts $H^{(1)}$ and $V-H^{(1)}$ does not introduce higher singularities than those of Coulomb types. Thus our result proves to be useful in completing the theory of the one-particle model of atomic systems.

12. Stability of the Coulomb potential to cut-off. In atom mechanics the Coulomb potential is usually assumed to be correct as far as the force-centers. For physical reasons, however, it would be necessary to "cut-off" the potential in some way or other. There arises the question whether the manner of cut-off affects the results seriously.

By means of the mathematical tools developed in this paper, we can show that the eigenvalues and eigenfunctions of the Hamiltonian operator with cut-off potential converge to those of the operator without cut-off when the cut-off distance tends to zero, and hence conclude that the cut-off does not affect the results appreciably provided that the cut-off distance is sufficiently small. We can say that the Coulomb potential is stable against cut-off procedure, which is a property not necessarily shared by all potentials. In this sense the Coulomb potential can be regarded as a faithful and convenient approximation to the real potential in atomic systems.

Acknowledgment. The writer wishes to thank Professor K. Ochiai for his interest in this work and Professor K. Kodaira for valuable instructions. His thanks are also due to Professor E. C. Kemble of Harvard University whose kindness made possible the publication of the present work, which was completed in 1944 but remained unpublished.

UNIVERSITY OF TOKYo, TOKYO, JAPAN 